\title{
Der Totalpbergsturz bei Davos aus bodenkundlicher Sicht
}

\section{Einleitung}

Der Totalpbergsturz bei Davos hat in letzter Zeit wieder vermehrt Anlaß zu Diskussionen gegeben, nachdem erkannt wurde, da $\beta$ es sich beim Absturz mindestens um ein zweiphasiges Ereignis gehandelt haben muß.

STRECKEISEN $(1974,1975,1981)$ nimmt zwei Bergsturzereignisse an. Die erste Sturzmasse bilde den Hügel des Drussetschawaldes und bestehe aus Kristallin der Davoser-Dorfbergdecke (Drussetscha-Bergsturz). Dieser soll nachträglich von einem zweiten Bergsturz (Wolfgang-Bergsturz) locker mit Serpentinit überdeckt worden sein. Von dieser zweiten Bergsturzmasse habe ein Teil als Murgang das Tal von Unter-Laret Richtung Klosters aufgefüllt (Abb.1). MAISCH (1981) ist dagegen der Meinung, daß das Gebiet Großweid/Stützwald durch sekundäres Abrutschen der nördlichen Teile der Drussetscha-Trümmer entstanden sei. Detaillierte Angaben über die verschiedenen Bergsturztheorien finden sich bei VUAGNEUX (1983). MAISCH (1981) schätzt das Alter des Bergsturzes auf etwa 14000 Jahre BP. Er ist also ins Spätglazial zu setzen, scheint aber nicht glazial überprägt zu sein (VUAGNEUX 1983). Diese Tatsache ist für den Bodenkundler äußerst interessant, hat er hier doch die Gelegenheit, die Bodenentwicklung auf reinem Serpentinitschutt ohne Beimischung von Moränenmaterial, wie das sonst in den Alpen der Normalfall ist, zu erforschen. Ziel der vorliegenden Arbeit war es, die Bodenbildung auf dem Bergsturzhügel östlich Wolfgang (Pt. 1631) zu untersuchen und anhand des vorgefundenen Bodenmusters die Bergsturztheorien zu überprüfen und allenfalls zu verfeinern.

\section{Untersuchungsgebiet}

Das Untersuchungsgebiet liegt östlich Wolfgang bei Davos (Kt. Graubünden) und umfaßt das etwa $1,9 \mathrm{~km}^{2}$ große Waldstück mit den Flurnamen: Delenwald, Budlerboden, Drussetschawald und Lusiwald (Abb.1: Karteriausschnitt der Landeskarte 1:25000, Blatt 1197 Davos).

\section{Methoden}

Mit der Hilfe von Stereoaufnahmen der Eidg. Landestopographie (Abb.7) wurde das Gebiet in Flächen gleicher Vegetationsbedeckung, beziehungsweise gleichen Reliefs, eingeteilt. Anschließend wurden in den diversen Flächen insgesamt 27 Bodenprofile gegraben und diese mittels Feldmethoden beschrieben. Die Farbe der Horizonte wurde nach den Revised Standard Color Charts (OYAMA \& TAKeHARA 1970) geschätzt. Der pH-Wert wurde an der getrockneten Probe im Labor gemessen $\left(0,01 \mathrm{M} \mathrm{CaCl}_{2}\right.$-Lösung, Boden: Lösung $1: 2,5$ ).

Vor der Aufnahme von Röntgendiffraktogrammen und IR-Spektren wurden die Proben zur Entfernung von freiem Eisen mittels Dithionit-Citrat behandelt.

Die Zuordnung der vorgefundenen Bodentypen zu den anhand des Luftbildes ausgeschiedenen Flächeneinheiten, sowie die Grenzbereinigung, erfolgte mittels Bohrstockaufnahmen. Wegen der großen Heterogenität der Böden konnten allerdings in vielen Fällen nicht bodentypreine Flächen, sondern lediglich Komplexe ausgeschieden werden.

Für die Erstellung der Bodenkarte diente die nicht entzerrte Vergrößerung des Luftbildes der Eidg. Landestopographie als Vorlage. Das Original weist einen ungefähren Maßstab von 1:12500 auf.

\section{Resultate und Diskussion}

\subsection{Die Böden auf dem Bergsturzkegel}

Abbildung 2 zeigt das Leitprofil für die Böden auf saurem Silikat im Drussetschawald. Charakteristisch ist der Bleichhorizont mit freigelegtem Quarz, unter welchem ein Humus- und Eisenanreicherungshorizont folgt. Diese Horizontabfolge ist typisch für einen Eisen-Humus-Podsol. Der Podsol bildet auf saurem Silikat, in der subalpinen Stufe unter Fichtenwald, den Klimaxboden. Im Drussetschawald findet man ihn meist zwischen großen Blöcken, wo Feinerde vorhanden ist. Die Blöcke selber sind von einer dicken Rohhumuslage überzogen. Hebt man die Rohhumusschicht von der Gesteinsunterlage ab, kommt die

Stephan Juchler, Dipl. Natw. ETH, H. Sticher, Prof. Dr.

Labor für Bodenkunde, ILW, ETH-Zentrum, 8092 Zürich 


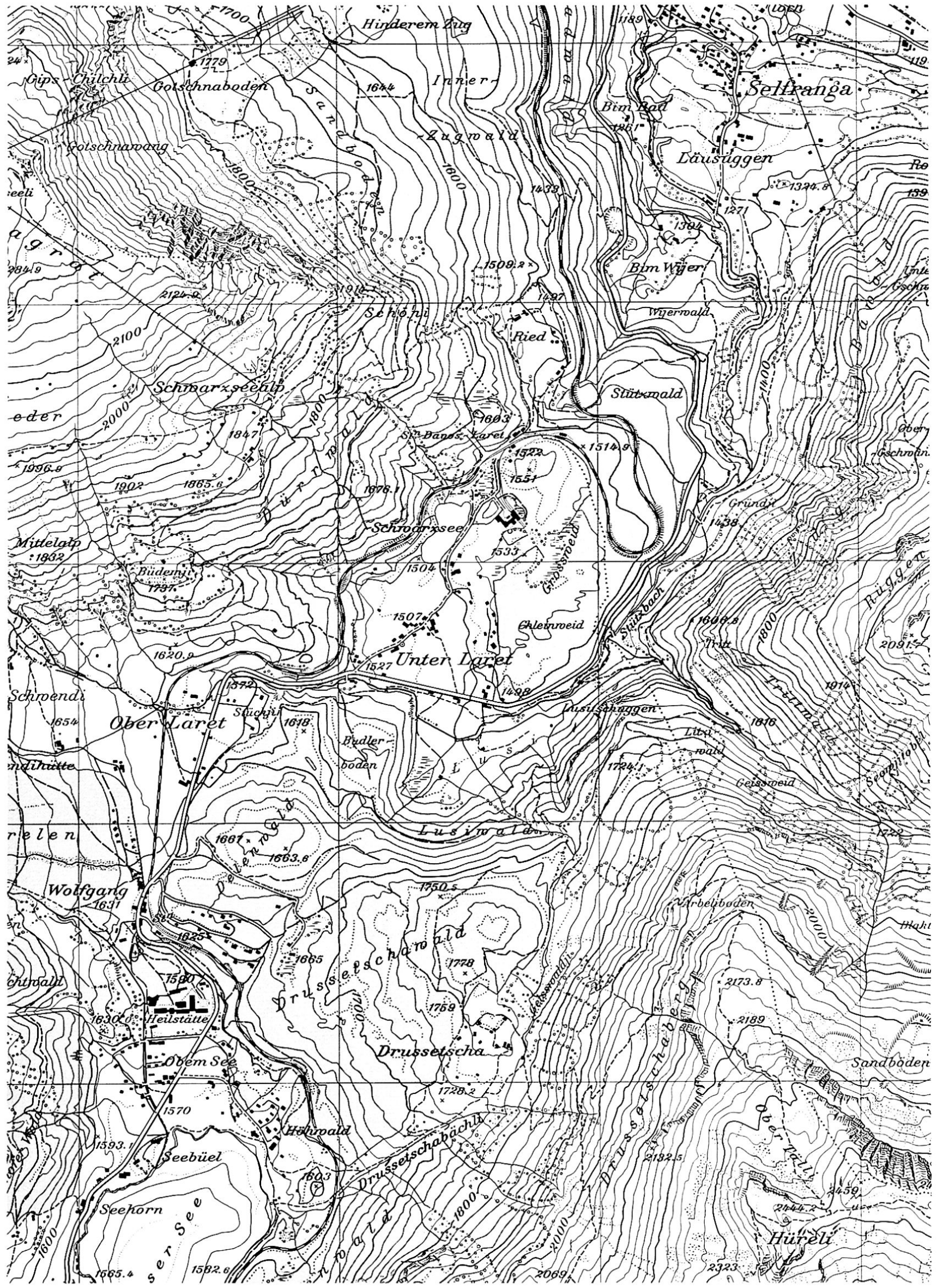

Abb.1 Ausschnitt aus der Landeskarte 1:25000 vom Untersuchungsgebiet (Reproduziert mit Bewilligung des Bundesamtes für Landestopographie vom 7.2.1985). 


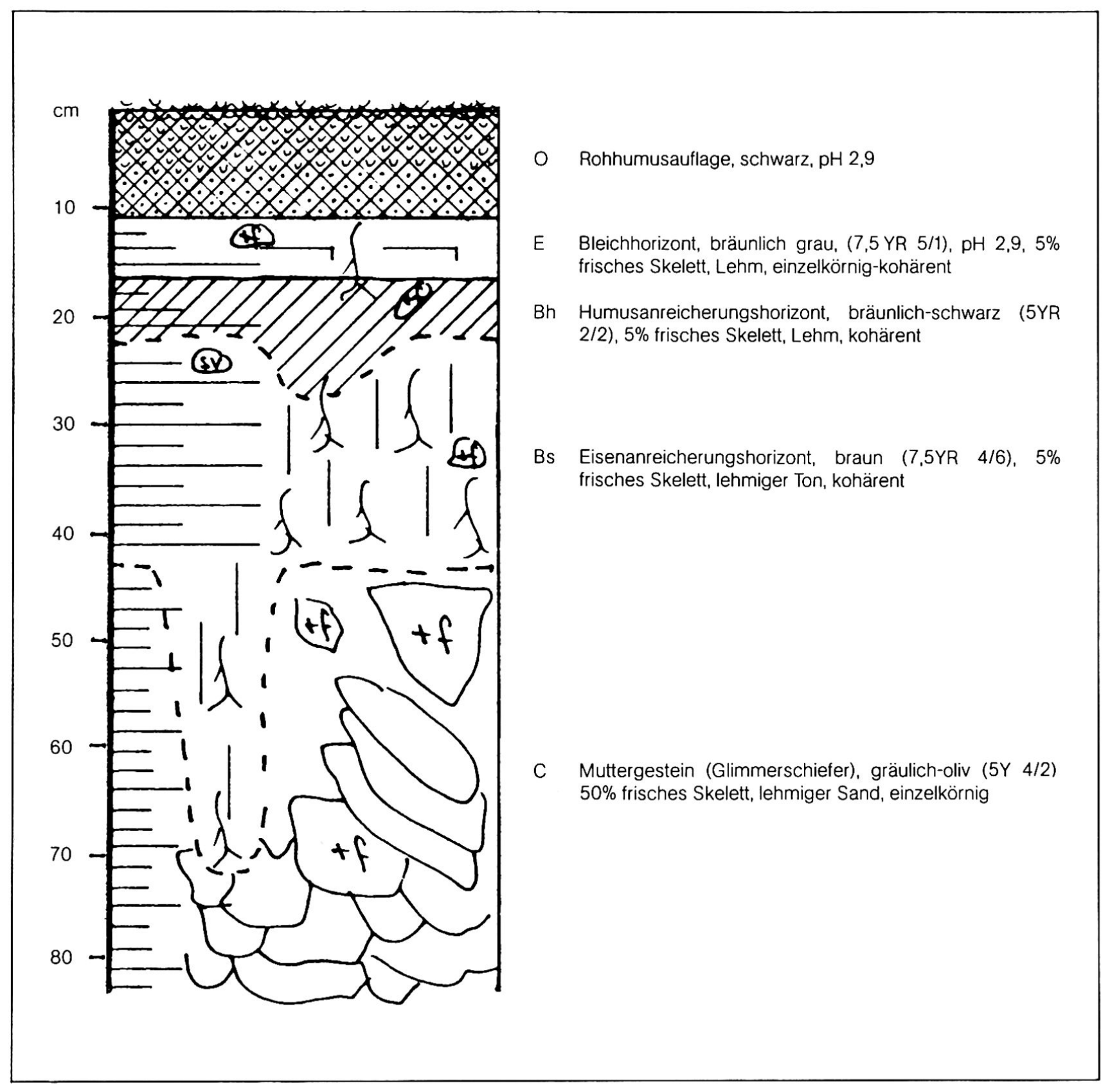

Abb. 2 Eisen-Humus-Podsol auf saurem Silikatblockschutt (Drussetschawald)

unverwitterte oft gebleichte Gesteinsoberfläche zum Vorschein. Einen solchen Bodentyp kann man als Rohhumus-Lithosol bezeichnen. BOCHTER (1984) schlägt dafür den Namen Ranpetrosol vor.

Ganz anders präsentieren sich die Böden im Delenwald, einem Erika-Bergföhrenwald auf Serpentinitblockschutt (Abb. 3). Unter der Humusauflage liegt ein tiefbrauner Verwitterungshorizont, der allmählich ins unverwitterte Muttergestein übergeht, das aber bis weit hinunter von eingewaschenem Feinstmaterial braun überzogen ist.

Die Humusauflage ist weniger mächtig als auf saurem Silikat, was auf die geringe organische Produktion der Böden auf Serpentinit zurückgeführt wird. Gründe für die spärliche Fruchtbarkeit dieser Böden sind vermutlich das ungünstig hohe $\mathrm{Mg} / \mathrm{Ca}$-Verhältnis, die geringen $\mathrm{K}$ - und $\mathrm{P}$-, sowie die hohen $\mathrm{Ni}$ - und $\mathrm{Cr}$-Gehalte des Gesteins (PROCTOR \& WOODELL 1975). Zusätzlich erfolgt der Abbau der organischen Substanz wegen der höheren pH-Werte schneller als auf saurem Silikat. Obschon Quarz im Muttergestein (Augitserpentinit nach PETERS 1963) fehlt, findet man gelegentlich unter der Humusauflage eine schwach ausgeprägte Bleichung der Mineralerde. Auf dieses Phänomen wird weiter unten eingegangen.

In Serpentinitböden kommt es gewöhnlich nicht $\mathrm{zu}$ einer sichtbaren Eisenverlagerung, weil die Huminstoffe durch die hohe Eisen- und Magnesiumkonzen- 


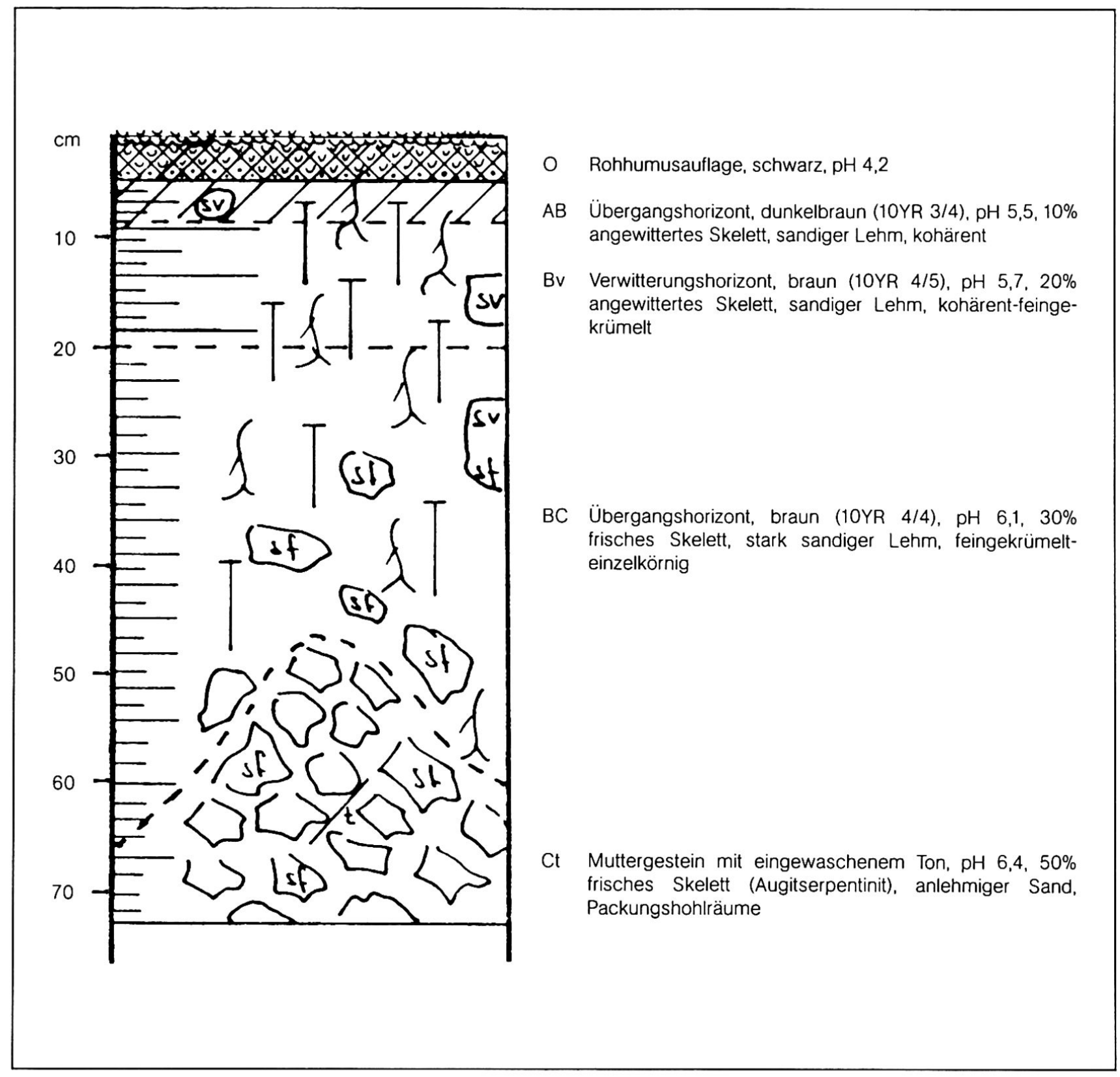

Abb. 3 Saure Serpentin-Braunerde auf Blockschutt (Delenwald)

tration rasch ausgefällt werden. $\mathrm{Da} ß$ hingegen Feinstmaterial leicht verlagert werden kann, wird auf den für die Tonverlagerung günstigen $\mathrm{pH}$-Wert von 5-6 im Unterboden und auf die durch Packungshohlräume bedingte große Porosität zurückgeführt. Als Namen für diese Böden schlagen wir saure Serpentin-Braunerde vor.

Im Drussetschawald findet man häufig Böden auf Mischgesteinschutt, bestehend aus Serpentinit und saurem Kristallin. Sie sind locker bestockt mit Fichten und Lärchen, gelegentlich auch Bergföhren. Je nach Gesteinstyp, der an der Oberfläche vorherrscht, geht die Bodenentwicklung Richtung Podsol oder Braunerde. Abbildung 4 zeigt einen Boden, dessen Muttergestein im Oberboden überwiegend aus saurem
Kristallin besteht. In $70 \mathrm{~cm}$ Tiefe wechselt die Farbe deutlich zu oliv-schwarz, der Farbe von frischem Serpentinitschutt. Trotz hohem Anteil von saurem Silikat kam es hier aber nicht zu einer Podsolierung, da wegen der Verwitterung des beigemischten Serpentinits der pH-Wert im Oberboden dafür zu hoch ist. Anhand der vorgefundenen Merkmale kann der Boden als saure Braunerde bezeichnet werden.

Im Steilhang des Lusiwaldes überwiegt als Muttergestein saures Kristallin. Infolge Erosion und Bodenrutschen sowie einer Hochstaudenflur, die hier von den feuchten Bedingungen des Nordhanges profitiert, kommt es nicht zu einer Podsolierung sondern zu einer Verbraunung (Abb. 5). Durch die gut abbaubare Streu und deren Vermischung mit dem Boden durch 


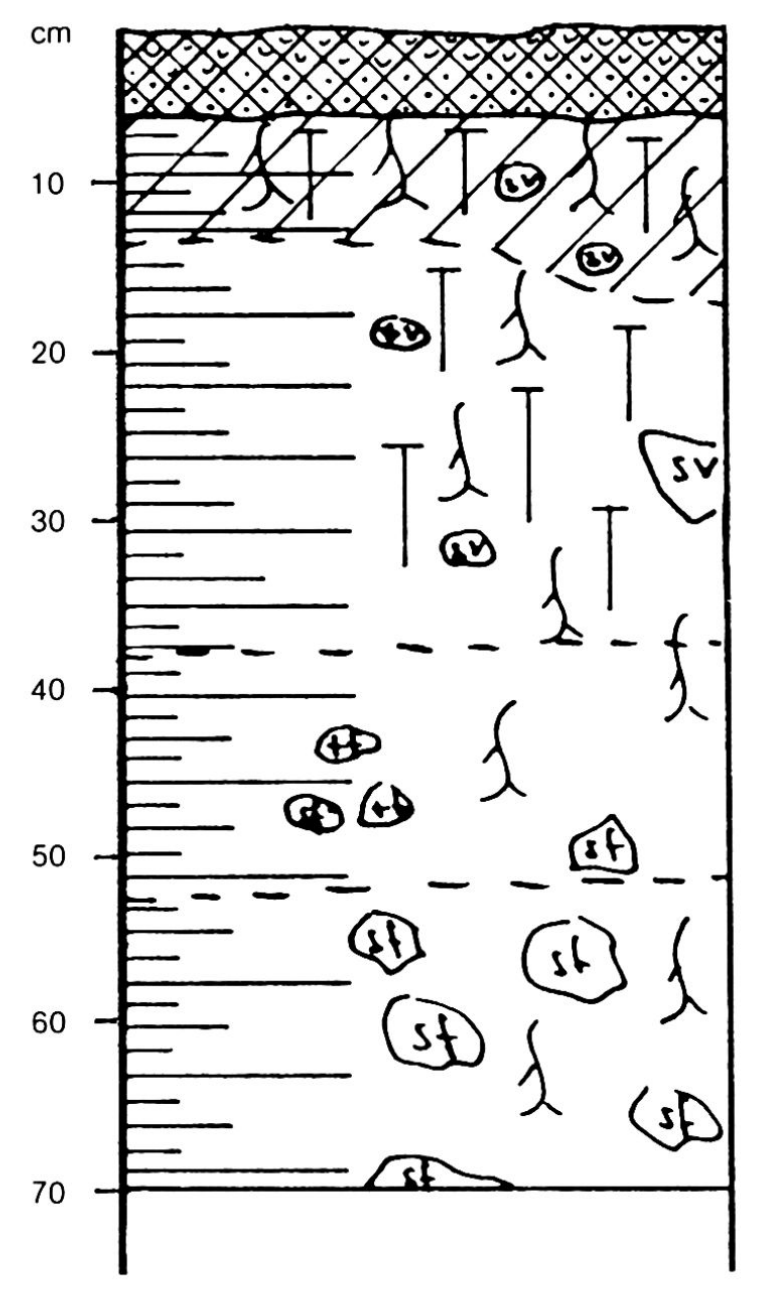

O Rohhumusauflage, schwarz, $\mathrm{pH} 3,9$

$A B$

Übergangshorizont, dunkelbraun (10YR 3/3), pH 5,1, 10\% angewittertes Skelett, Lehm, kohärent

Bv Verwitterungshorizont, braun (10YR 4/4), pH 5,5, 10\% angewittertes Skelett, Lehm, kohärent

C Muttergestein (20\% Serpentinit, Rest saures Silikat), gräulich-oliv (5Y 5/3), pH 5,9,10\% frisches Skelett, sandiger Lehm, kohärent

IIC Serpentinitschutt mit weniger als $10 \%$ saurem Silikat, olive-schwarz (5Y 3/2), pH 6,2, 25\% frisches Skelett, sandiger Lehm, kohärent

Abb. 4 Saure Braunerde auf Mischgesteinsschutt (Drussetschawald)

Hangrutschung entsteht ein Mull. Die Rutschung bewirkt im weiteren eine hangparallele Einregelung der Steine und eine ungleichmäßige Ausbildung der Horizonte. Wir bezeichnen diesen Boden als HangBraunerde.

In Senken des Drussetschabergsturzhügels findet man vereinzelt auch hydromorphe Böden. Sie sind aber flächenmäßig unbedeutend.

\subsection{Bleichhorizont in Serpentinitböden}

Wie bereits erwähnt, stößt man im Delenwald auf Bleichhorizonte (Abb.6), obschon das Muttergestein (reiner Serpentinitschutt) frei von Quarz ist. Die IR-Spektren und Röntgendiffraktogramme zeigen aber eindeutig Quarz an, mit Spuren von Feldspat, Glimmer und anderen Mineralien. Serpentin hingegen fehlt. STICHER (1981) hat im Verwitterungshorizont von Serpentinbraunerden Quarz mit einem Maximum in der Korngrößenverteilungskurve um 20 $\mu \mathrm{m}$ bestimmt. Wir schließen daraus, da $\beta$ dieses Material aus Flugstaub stammen muß.

Das ungleichmäßige Vorkommen dieses Bleichhorizontes kann vom Großrelief bestimmt sein, indem je nach Exposition verschieden stark podsolierende Bedingungen herrschen, die erst den Quarzstaub sichtbar machen können. Andererseits kann das Kleinrelief (die Bodenoberfläche ist durch die vielen Steinblöcke sehr unregelmäßig ausgebildet) für die Ablagerung und Akkumulation von Flugstaub unter- 


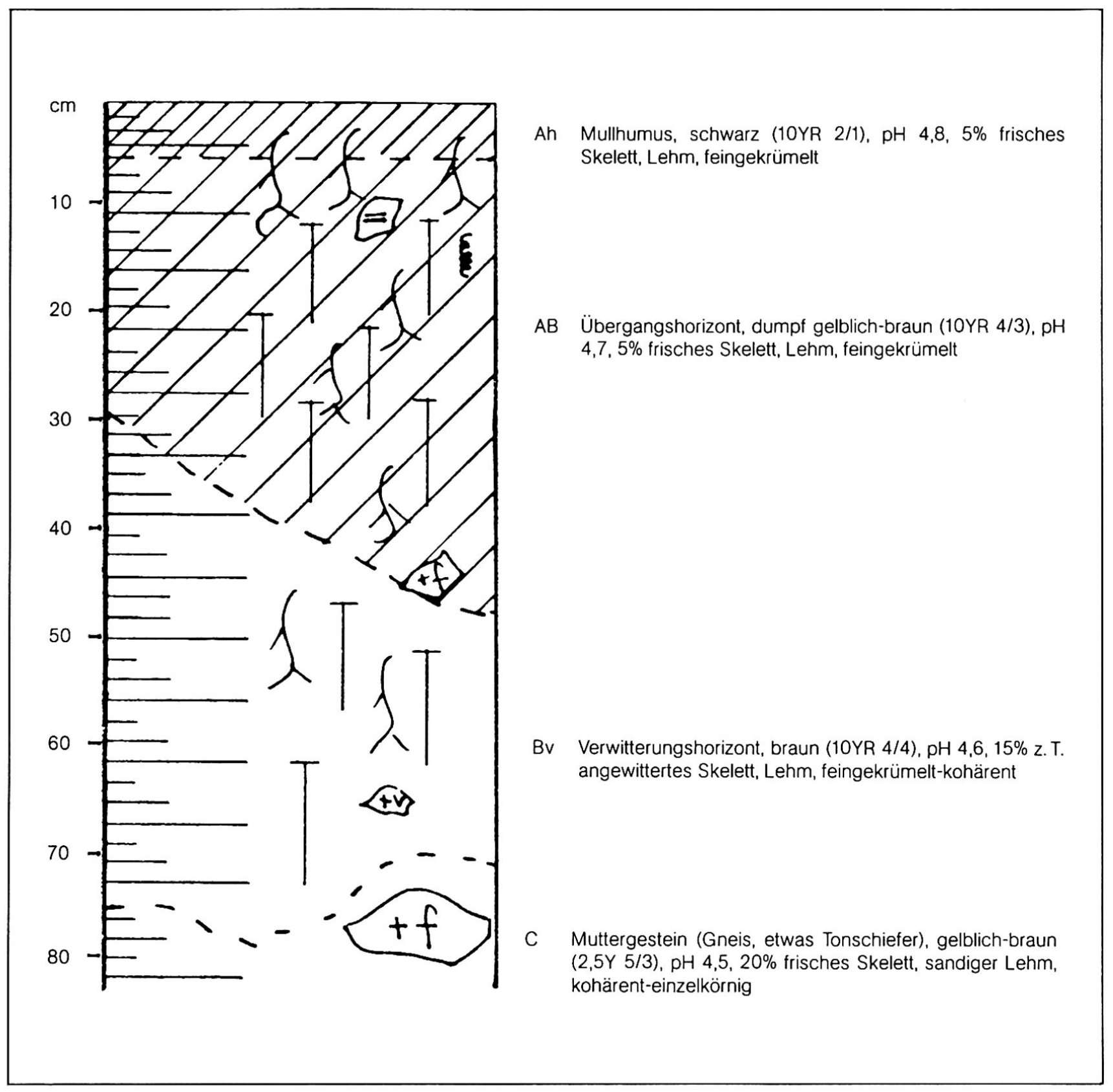

Abb. 5 Mull-Hangbraunerde auf saurem Silikatschutt (Lusiwald)

schiedliche Bedingungen geschaffen haben. An einem Hügel konnten wir zeigen, daß das $\mathrm{pH}$ des 0 -Horizontes am Nordhang tiefer ist als am Südhang; nämlich 3,7 respektive 4,1. Ferner stießen wir mit dem Bohrstock am Nordhang mit einer Wahrscheinlichkeit von $44 \%$ auf eine Bleichung der obersten Mineralschicht gegenüber $14 \%$ am Südhang. Diese Unterschiede sind auf dem $90 \%$-Niveau signifikant.

\subsection{Bodenkarte}

Auf der Bodenkarte (Abb. 8) fallen die im Nordwesten gelegenen, als homogene Flächen ausgeschiedenen, horizontal-linierten, Delenwald und Budlerboden auf.

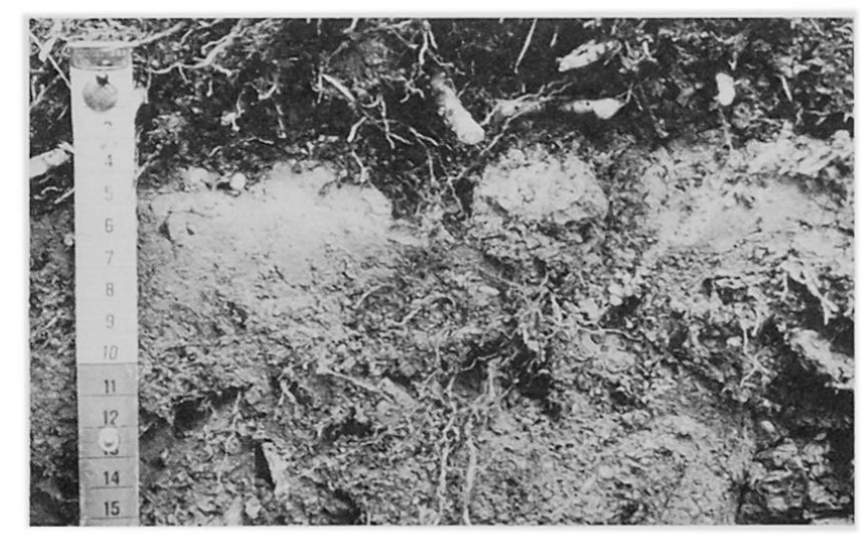

Abb. 6 Serpentin-Braunerde mit Bleichhorizont 


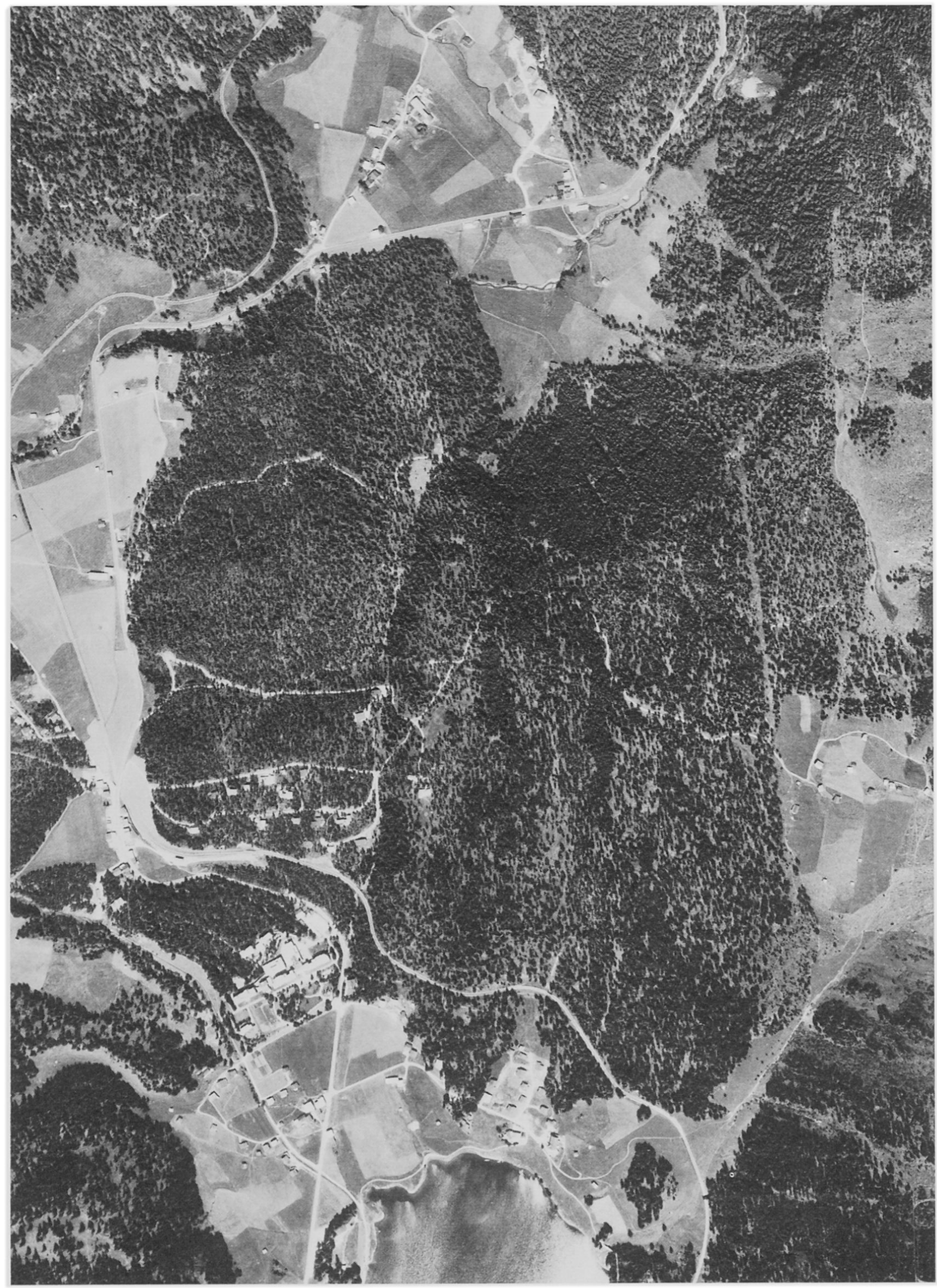

Abb. 7 Luftaufnahme des Untersuchungsgebietes (Reproduziert mit Bewilligung des Bundesamtes für Landestopographie vom 7. 2.1985). 

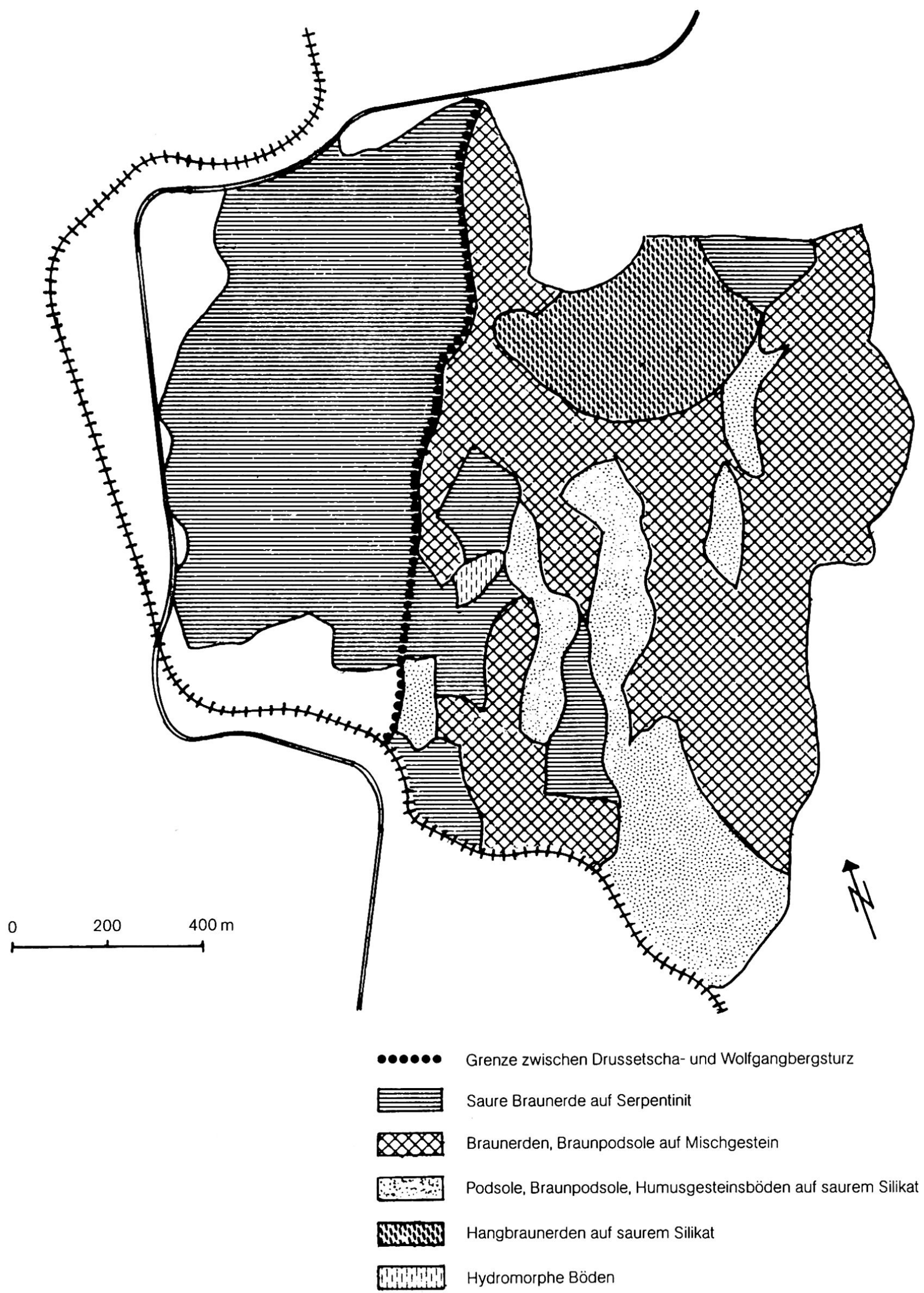

Abb. 8 Bodenkarte des Untersuchungsgebietes 
In diesem Gebiet tritt als Muttergestein reiner blockiger Serpentinitschutt auf. Als Bodentyp findet man saure Serpentin-Braunerden, die unter Humusauflage teilweise einen dünnen Bleichhorizont aufweisen.

Im Drussetschawald markieren die punktierten Flächen den Bodenkomplex Podsol (Eisen-Humus-Podsole, Braunpodsole, Humus-Gesteinsböden) auf reinem sauren Kristallin. Dieses Substrat ist meist sehr grobblockig (bis hausgroß) und mit dichtem Fichtenwald bedeckt.

Die karierten Flächen tragen demgegenüber vorwiegend einen lockeren Lärchen-Fichtenwald und wurden wahrscheinlich früher, zum Teil auch heute noch, beweidet. Das Substrat ist feinkörniger als dasjenige im Delenwald und besteht aus einem Gemisch von Serpentinit und saurem Kristallin, alternierend oder vermischt.

Das Muttergestein im Lusiwald besteht fast ausschließlich aus saurem Kristallin (Gneise, Tonschiefer) wenig Serpentinit und einigen carbonathaltigen Steinen. Auf den dort vorkommenden Hangbraunerden stockt ein dichter Fichtenwald. Ganz im östlichen Teil des Lusiwaldes tritt wieder fast reiner Serpentinitschutt mit Serpentinbraunerde zutage.

\subsection{Der Bergsturz aus bodenkundlicher Sicht}

Die Bodenkarte bestätigt die Theorie von den zwei hintereinanderfolgenden und aus verschiedenen $\mathrm{Ge}$ steinen bestehenden Bergstürzen (STRECKEISEN 1974). Der Drussetscha- und Lusiwald mit dem Gemisch von saurem Kristallin und Serpentinit können dem Drussetschabergsturz, der Delenwald und Budlerboden mit reinem Serpentinit dem Wolfgangbergsturz zugeordnet werden. Die Grenze zwischen den beiden Ablagerungsgebieten zieht sich als deutliche Linie im Gelände von der Heilstätte im Süden zum Budlerboden im Norden (Abb.7). Dort ist dann allerdings die Grenzziehung etwas schwieriger. Wahrscheinlich verläuft die Grenze auf der östlichen Krete des Budlerbodens, da diese zum Teil aus saurem Kristallin besteht, während westlich davon nur noch reiner Serpentinitschutt vorkommt.

Wie STRECKEISEN (1975) beobachtete, besteht die Hauptmasse des Drussetschabergsturzes aus saurem Kristallin. Der Lusiwald zeigt gewissermaßen einen Aufschluß dieses Bergsturzkegels. Wir gehen aber mit der Ansicht von STRECKEISEN (1974), wonach der Drussetschabergsturzhügel erst nachträglich von Serpentinit überschüttet worden wäre, nicht einig. Wir vermuten vielmehr, daß schon mit dem 1 . Bergsturz Serpentinit mitgeführt worden ist. Das zeigen die Mischgesteinsböden im Drussetschawald, die zum Teil eine innige Vermischung der beiden Gesteinstypen, und zum Teil sogar eine Überlagerung des Serpentinites mit saurem Silikat aufweisen. Ferner scheint es unwahrscheinlich, daß Serpentinitschutt den Drussetschabergsturzhügel überschüttet haben sollte, ohne in den auf der Bodenkarte punktierten
Flächen, die quer zur Überschüttungsrichtung liegen, Spuren zu hinterlassen. Eine solche Überschüttung scheint uns nur am Westrand des Drussetschawaldes im Gebiet rund um Punkt 1665 wahrscheinlich. Daß beide Gesteine miteinander heruntergekommen sind, mit zum Teil unvollständiger Durchmischung, zeigt der östlichste Teil des Lusiwaldes, wo wieder Serpentinitschutt liegt.

Die großen Blöcke im Drussetschawald gaben verschiedentlich Anlaß zu Vermutungen über einen zusätzlichen Bergsturz vom Hüreli, also vom Hang östlich der Drussetschaalp (MAISCH 1981). Tatsächlich sieht man am Drussetschaberg eine Ausbruchsnische. Es scheint aber, daß praktisch alles herausgebrochene Material am Unterhang selber liegen blieb und nur vereinzelte Blöcke auf den Bergsturzhügel bis in den Raum der heutigen Drussetschaalp, weiterrollten. Dieser Ansicht ist auch STRECKEISEN (1981, 1984: persönliche Mitteilung), der die großen Blöcke im Drussetschawald als Dorfbergkristallin identifiziert hat.

Der Komplex Großweid/Stützwald, den wir allerdings nicht detailliert kartiert haben, scheint zum Drussetschabergsturz zu gehören. Wie Bohrstockproben und Aufschlüsse zeigen, bestehen diese Massen aus einem Gesteinsgemisch und nicht aus reinem Serpentinit, wie das für den Wolfgangbergsturz typisch ist. Das würde die Theorie von MaISCH (1981) stützen, der im Lusiwald die Ausbruchnische eines sekundären Sturzes, der jetzigen Großweid/Stützwaldmassen, Richtung Klosters sieht.

Zusammenfassend kann man die Bergsturzereignisse aus der Sicht der Bodenkunde folgendermaßen darstellen:

1. Ein Bergsturz bestehend aus Dorfbergkristallin und Serpentinit füllt das ehemalige Landquarttal bei Wolfgang Richtung Klosters auf und bildet den Drussetschahügel.

2. Ein praktisch reiner Serpentinitbergsturz lagert sich an den 1. Bergsturzhügel an und bildet den Delenwaldhügel und den Budlerboden.

3. Der nördliche Teil des Drussetschahügels löst sich ab und bildet die Masse Großweid/Stützwald.

Über die zeitliche Einstufung dieser 3 Ereignisse kann aus der Sicht der Bodenkunde keine Aussage gemacht werden.

\section{Zusammenfassung}

Der Totalpbergsturzhügel östlich Wolfgang bei Davos wurde mittels 27 Profilen bodenkundlich untersucht und kartiert.

Auf Serpentit entwickelten sich trotz subalpiner Stufe im Gegensatz zu saurem Silikat keine Podsole, sondern Braunerden. Gelegentlich auftretende, schwach ausgeprägte Bleichhorizonte unter der Rohhumusschicht der Serpentinböden bestehen überwiegend aus Quarzschluff, der jedoch nicht autochthon, sondern äolischen Ursprungs ist. 
Die Kartierung macht die Grenze zwischen den von zwei verschiedenen Bergstürzen stammenden Ablagerungsgebieten deutlich. Der 1. Bergsturz (Ablagerungsgebiete: Drussetschawald und Lusiwald) bestand vorwiegend aus saurem Kristallin mit beigemischtem Serpentinit, während der nachfolgende Sturz (Delenwald, Budlerboden) aus reinem Serpentinit bestand und sich dem Vorgänger nur anlagerte oder ihn höchstens randlich überschüttete.

\section{Abstract}

The soils of a deposition hill of an ancient mountain slide, from a serpentinitic region called Totalp, were investigated and mapped. The investigation area lies east of Wolfgang (1631 m above sea level) near Davos, Switzerland.

In spite of the subalpine climate, which generates iron-humus-podsols on acid silicates, acid brown earth was found on serpentinitic parent material. Slightly visible eluvial horizons that were found occasionally below the raw humus consisted primarily of quartzsilt, which was thought to be of aeolian origin.

Mapping elucidated the boundary between two mountain slide depositions. The first slide to occur (deposition area: Drussetschawald/Lusiwald) consisted mainly of acid silicates mixed with some serpentinite, whereas the slide that followed (Delenwald/Budlerboden) consisted of pure serpentinite. This second slide only covered the first marginally.

\section{Literatur}

BOCHTER R. (1981): Bodenbildung auf Kalk- und Gneisbergsturzblöcken unter subalpinem Fichtenwald. Z. Pflanzenernähr. Bodenk. 147, 604-613.

MAISCH M. (1981): Glazialmorphologische und gletschergeschichtliche Untersuchungen im Gebiet zwischen Landwasserund Albulatal (Kt.Graubünden, Schweiz). Diss. Juris Druck \& Verlag, Zürich, $215 \mathrm{~S}$.

OYAMA M. und TAKEHARA H. (1970): Revised Standard Soil Color Charts. 2. Auflage, Japan.

PETERS T. (1963): Mineralogie und Petrographie des Totalpserpentins bei Davos. Diss. Dissertationsdruckerei Leemann AG, Zürich, $154 \mathrm{~S}$.

PROCTOR J. und WOODELL S. (1975): The Ecology of Serpentine Soils. Adv. Ecol. Res. 9, 256-366.

STICHER H. (1981): Die Böden auf dem Totalpserpentin bei Davos. In: Bodenkundliche Exkursionen in den Kanton Graubünden 28./29. August 1981. Bodenkundliche Gesellschaft der Schweiz, 24-38.

STRECKEISEN A. (1974), unpubl.: Aufnahmebericht an die Geologische Kommission.

STRECKEISEN A. (1975), unpubl.: Aufnahmebericht an die Geologische Kommission.

STRECKEISEN A. (1981): Schriftliche Mitteilung an Dr. Max Maisch, Geographisches Institut der Universität Zürich.

VUAGNEUX R. (1983): Glazialmorphologische und gletschergeschichtliche Untersuchungen im Gebiet Flüelapaß. Physische Geographie 10, Zürich, 249 S.
TESDORPF Jürgen C.: Landschaftsverbrauch. $586 \mathrm{~S}$., 34 Abb., 94 Tab., Verlag Tesdorpf, Vilseck, 1984, DM 38.--.

Landschaftsverbrauch ist wohl eines der aktuellsten, aber auch schwierigsten Themata (abgesehen vom Egoismusproblem). Dies verrät auch das Buch des langjährigen Regionalplaners, zumal mit seiner rund 4000 Titel zählenden Bibliographie. Bereits die Definition von Landschaftsverbrauch verursacht beinahe unlösbare Probleme. Tesdorpf anerkennt Landschaft als Realität; er sucht über sie eine Lösung des Begriffs ihres Verbrauchs, den er unter Hinweis auf die semantische Forschung ironisch als "Sprengsatz" sachlich als "Komplex aus quantitativem Freiflächenentzug und qualitativen Negativwirkungen auf die Landschaft" umschreibt. In der Folge wird er "am Beispiel Baden-Württembergs empirisch belegt und seine sektoralen und regionalen Aspekte aufgezeigt". Er betrug, kursorisch zusammengefasst, 1950-78 15,6 ha (Schweiz 1941-67 Kulturlandverlust 9,7 ha). Eine Expertenumfrage bei Wissen- schaftern, Stadt- und Regionalplanern und Politikern diente dazu, Aufschlüsse über die Ursachen zu gewinnen. Mit Hilfe multivariater statistischer Verfahren wurde das Phänomen gesichert. Teil $C$ des Buches schliesslich, die besonderen Planungsprobleme enthaltend, gibt über die Möglichkeiten Aufschluss, den Verbrauch zu "stoppen", zu "verlangsamen",zu "bremsen" oder zu "verringern" (mit über 300 Massnahmen). "Besonderer Wert (wurde) darauf gelegt, dass die Vorschläge in verschiedenen Lebensbereichen (Gesetzgebung, Politik, Planung, Verwaltungsvolizug, Wissenschaft) ansetzen. Ein "Massnahmennetz" im Anhang ist das "Konzentrat" der Untersuchung. Sie ist als Ganzes - jeder Abschnitt verdiente gesonderte längere Würdigung - die mustergültige Darlegung eines komplexen Gegenwarts- und $\mathrm{Zu-}$ kunftsproblems, das "brennt" und die deshalb jedem "theoretischen" und "praktischen" Geographen zu gründicher Stellungnahme empfohlen sei.
GH $3 / 85$
E. Winkler, Zürich 